\title{
Interactions between hematopoietic progenitor kinase 1 and its adaptor proteins (Review)
}

\author{
QING ZHANG ${ }^{1}$, SHU DING ${ }^{2}$ and HUILIN ZHANG ${ }^{3}$ \\ ${ }^{1}$ Department of Dermatology, Second Xiangya Hospital, Central South University, Changsha, Hunan 410011; \\ ${ }^{2}$ Department of Dermatology, Third Xiangya Hospital, Central South University, Changsha, Hunan 410013; \\ ${ }^{3}$ Emergency Department, Second Xiangya Hospital, Central South University, Changsha, Hunan 410011, P.R. China
}

Received January 4, 2017; Accepted July 31, 2017

DOI: $10.3892 / \mathrm{mmr} .2017 .7494$

\begin{abstract}
Hematopoietic progenitor kinase 1 (HPK1), also known as mitogen-activated protein kinase kinase kinase kinase 1 is a serine/threonine protein kinase. It is involved in various cellular events, including mitogen-activated protein kinase signaling, nuclear factor- $\kappa \mathrm{B}$ signaling, cytokine signaling, cellular proliferation and apoptosis, $\mathrm{T}$ cell receptor/B cell receptor signaling and $\mathrm{T} / \mathrm{B} / \mathrm{dendritic}$ cell-mediated immune responses. Therefore, HPK1 has variety of roles in immunity, and is associated with the pathogenesis of autoimmune diseases, cancer, and the inflammatory response. In these cellular and immune events, HPK1 interacts with several adaptor proteins, including caspase recruitment domain family, member 11 , hematopoietic cell-specific protein 1, HPK1-interacting protein of $55 \mathrm{kDa}$, the growth factor receptor-bound protein 2 family, linker for activated T-cells, the SH2 domain-containing leukocyte protein of $76 \mathrm{kDa}$ family, the v-crk avian sarcoma virus CT10 oncogene homolog family, B-cell adaptor molecule of $32 \mathrm{kDa}$ and non-catalytic region of tyrosine kinase adaptor protein. These adaptor proteins can couple HPK1 with various effector molecules, leading to the transmission of upstream signals to downstream targets. They are crucial in regulating the relocation, phosphorylation, activation and functions of HPK1. HPK1 can also phosphorylate certain proteins, consequently modulating their functions. This review aims to describe the adaptor proteins, which interact with HPK1, particularly focusing on their modes of interaction with HPK1, and the effects that these interactions cause.
\end{abstract}

Correspondence to: Dr Huilin Zhang, Emergency Department, Second Xiangya Hospital, Central South University, 139 Renmin Middle Road, Changsha, Hunan 410011, P.R. China

E-mail: 245145077@qq.com

Key words: hematopoietic progenitor kinase 1, mitogen-activated protein kinase kinase kinase kinase 1, adaptor protein, T cell receptor, B cell receptor

\section{Contents}

1. Introduction

2. Roles of HPK1 in immunity

3. Adaptor proteins

4. CARD11

5. HS1

6. HIP-55

7. GRB2 family

8. LAT

9. SLP-76 family

10. CRK family

11. BAM32

12. NCK

13. Conclusions and future directions

\section{Introduction}

Hematopoietic progenitor kinase 1 (HPK1), also known as mitogen-activated protein kinase kinase kinase kinase 1 (MAP4K1) is a $97 \mathrm{kDa}$ serine/threonine protein kinase, which belongs to the germinal center kinase family $(1,2)$. It is comprised of an N-terminal Ste20-like kinase domain and four proline-rich regions (P1, P2, P3 and P4, respectively). P1, $\mathrm{P} 2$ and $\mathrm{P} 4$ contain the typical class II binding motif, which can bind adaptor proteins containing Src homology 3 (SH3) domains (3-5). HPK1 also has a citron homology (CNH) domain at its C-terminus, which may act as a regulatory domain and is involved in molecular interactions (6-8) (Fig. 1). It contains 13 potential tyrosine phosphorylation residues, a number of which can be phosphorylated by $\xi$-chain associated protein kinase $70 \mathrm{kDa}$ (ZAP-70), providing potential docking sites for Src homology 2 (SH2)-containing molecules (9).

The activation ofHPK1 isinvolved in adaptorprotein binding, relocating to the plasma membrane, autophosphorylation and transphosphorylation by protein kinase D1 (a serine/threonine kinase) and protein tyrosine kinases (PTKs) $(4,8,10,11)$. It can be activated by a variety of stimuli $(2,12)$, including epidermal growth factor $(\mathrm{EGF})(13,14)$, prostaglandin $\mathrm{E}_{2}\left(\mathrm{PGE}_{2}\right)(15)$, transforming growth factor $\beta$ (TGF- $\beta$ ) $(16,17)$, erythropoietin (EPO) (18), T cell receptor (TCR) and B cell receptor (BCR) stimulation $(2,4,7,19)$. During T cell activation, HPK1 functions upstream of protein kinase C (PKC) and Ras (6). 


\section{Roles of HPK1 in immunity}

HPK1 has a variety of roles in immunity. As a serine/threonine protein kinase, HPK1 serves as an MAP4K and can transmit signals through mitogen-activated protein kinase kinase kinase (MAP3K), including mitogen-activated protein kinase kinase (MEKK) 1, mixed-lineage protein kinase 3 and TGF- $\beta$ activated kinase-1 (TAK1) to mitogen-activated protein kinase kinase (MAP2K), including MAPK kinase (MKK) 4 and MKK7, finally resulting in the activation of c-Jun N-terminal kinase (JNK) (16,17,20-22).

HPK 1 can activate the $\alpha$ and $\beta$ subunits of the inhibitor of nuclear factor- $\kappa \mathrm{B}(\mathrm{I} \kappa \mathrm{B})$ kinase (IKK) complex, resulting in $\mathrm{I} \kappa \mathrm{B}$ degradation and nuclear factor- $\mathrm{\kappa B}(\mathrm{NF}-\kappa \mathrm{B})$ activation (23).

HPK1 is cleaved into two fragments by caspase- 3 during apoptosis at Asp385 within a conserved DDVD ${ }^{385}$ motif: An $\mathrm{N}$-terminal region (43 kDa; aa2-aa385), which contains the kinase domain and $\mathrm{P} 1$, and a $\mathrm{C}$-terminal region $(54 \mathrm{kDa}$; aa386-aa833), which contains the CNH domain and the other three proline-rich regions $(8,19,24,25)$ (Fig. 1). The N-terminal domain of HPK1 retains the ability to activate JNK, whereas the C-terminal domain of HPK1 becomes an inhibitor of NF- $\kappa B(7,24,25)$. HPK1 also induces the expression of Fas ligand (FasL) in T cells $(2,26)$.

The JNK family is involved in various $\mathrm{T}$ cell responses, which include Th1/Th2 differentiation, cellular proliferation and apoptosis $(26,27)$. It has been shown that the activation of NF- $\kappa$ B can protect cells from cellular apoptosis $(23,28,29)$, whereas FasL is a known inducer of apoptosis in T cells $(26,28,29)$. The ability of HPK1 to regulate JNK, NF- $\kappa \mathrm{B}$ and FasL simultaneously indicates its complex and conflicting role in controlling cellular proliferation and apoptosis.

In addition to regulating cellular proliferation and apoptosis, HPK1 is a negative regulator of TCR/BCR signaling and T/B cell-mediated immune responses $(1,11,30-32)$. Shui et al found that $\mathrm{HPK}^{-/}$mouse $\mathrm{T}$ cells are hyperproliferative in response to stimulation with anti-CD3 and anti-CD28 antibodies, and these cells produce increased IL-2, IL-4 and interferon (IFN)- $\gamma$ when immunized with $\mathrm{T}$ cell-dependent antigens (6). In addition, $\mathrm{T}$ cell-dependent humoral responses in $\mathrm{HPK} 1^{-/}$mice are more marked, compared with those in controls and HPK $1^{-1-}$ mice have been shown to exhibit more severe autoimmune phenotypes in an experimental autoimmune encephalomyelitis model of multiple sclerosis in mice (6). As with T cells, HPK1 negatively regulates Ras-related protein 1 (RAP1)-mediated $\mathrm{B}$ cell adhesion; $\mathrm{HPK}^{-/}$mice B cells appear hyperresponsive to a range of stimuli (12).

In humans, HPK1 is important roles in the pathogenesis of systemic lupus erythematosus (SLE). It has been shown that the mRNA and protein levels of HPK1 decreased significantly in $\mathrm{CD} 4^{+} \mathrm{T}$ cells from patients with SLE, compared with those in healthy controls, and its mRNA expression was negatively correlated with disease activity in the patients with SLE. When the expression of HPK1 was knocked down in healthy $\mathrm{CD}^{+} \mathrm{T}$ cells using small interfering (si) RNA, increased $\mathrm{T}$ cell proliferation, overproduction of IFN $\gamma$ and excessive $\mathrm{B}$ cell stimulation was observed. By contrast, plasmid-induced overexpression of HPK1 in SLE CD4 ${ }^{+} \mathrm{T}$ cells resulted in reduced $\mathrm{T}$ cell proliferative activity, and decreased IFN $\gamma$ and $\mathrm{IgG}$ synthesis. Therefore, HPK1 may serve as a novel target in effective SLE therapy (31). In addition to SLE, microarray analyses have suggested that the level of HPK1 is downregulated in the peripheral blood cells of patients with psoriatic arthritis $(33,34)$.

In addition to autoimmune diseases, HPK 1 is involved in the pathogenesis of cancer. It has been found that the level of HPK1 is altered in acute myeloid leukemia $(35,36)$, bladder urothelial carcinoma (37), extramammary Paget's disease (38) and colon carcinoma (39). However, unlike in autoimmune diseases, the expression of HPK1 is increased in these types of cancer. Of note, it has been reported that inhibiting HPK1 promotes resistance to cancer, and this results from the role of HPK1 in immunity; HPK1 is a negative regulator of T cell and dendritic cell (DC) activation. Mature HPK $1^{-1-}$ bone marrow-derived DCs (BMDCs) are superior to their wild-type counterpart in stimulating $\mathrm{T}$ cell proliferation in vivo and in vitro. This is due to mature $\mathrm{HPK}^{-/-} \mathrm{BMDCs}$ expressing higher levels of costimulatory molecules CD80, CD86 and I- $\mathrm{A}^{\mathrm{b}}$, and higher levels of proinflammatory cytokines interleukin (IL)-1 $1 \beta$, IL-6, IL-12 and tumor necrosis factor (TNF)- $\alpha$. HPK $1^{-1-}$ BMDCs are significantly resistant to lipopolysaccharide-induced apoptosis. The vaccination of Lewis lung carcinoma-bearing mice with HPK $1^{-/-}$BMDCs can result in complete tumor eradication $(1,40)$.

Alzabin et al (11) demonstrated that $\mathrm{HPK}^{-/}$mouse T cells are resistant to TCR- and $\mathrm{PGE}_{2}$-induced apoptosis, and they can withstand $\mathrm{PGE}_{2}$-mediated suppression of $\mathrm{T}$ cell proliferation, producing increased IL-2 and IFN $\gamma$; following the transfer of a Lewis lung carcinoma tumor cell line (3LL) intravenously into wild-type or HPK $1^{-/}$mice, the number and size of tumors were smaller in the HPK $1^{-/}$mice, compared with those in wild-type counterparts, and this tumor inhibition was T cell-mediated. When HPK $1^{-/}$mice splenocytes were co-cultured with the $3 \mathrm{LL}$ cells, they were five times more effective in killing 3LL cells than the wild-type splenocytes. Following intravenous injection of wild-type or $\mathrm{HPK}^{-1-} \mathrm{T}$ cells with $3 \mathrm{LL}$ cells into T cell-deficient mice, the number and size of tumors were markedly reduced in the lungs of the mice exposed to HPK $1^{-/} \mathrm{T}$ cells. These results suggest that reducing the level of HPK1 leads to an enhanced anti-tumor response by $\mathrm{T}$ cells $(11,40)$.

HPK1 is also involved in the inflammatory response. It is important for chemokine (C-X-C motif) ligand 1-induced lymphocyte function associated antigen 1 (LFA-1)-mediated polymorphonuclear neutrophil (PMN) adhesion to intercellular adhesion molecule 1 (ICAM)-1 during the acute inflammation. In addition, HPK1 is involved in CD11/CD18-mediated adhesion strengthening, spread and the directed mechanotactic crawling of PMNs under flow conditions. PMN adhesion and extravasation are severely defective in HPK1-deficient mice (41).

Geisberger et al (42) reported that HPK1 is cleaved into $\mathrm{N}$ - and C-terminal fragments during monocytic differentiation, and the N-terminal region of HPK1 can induce the differentiation of progenitor cells towards the monocytic lineage (19). HPK1 interacts with the cytoplasmic tail of membrane IgE. This may represent a missing link to upstream regulatory elements in HPK1 activation (42). 


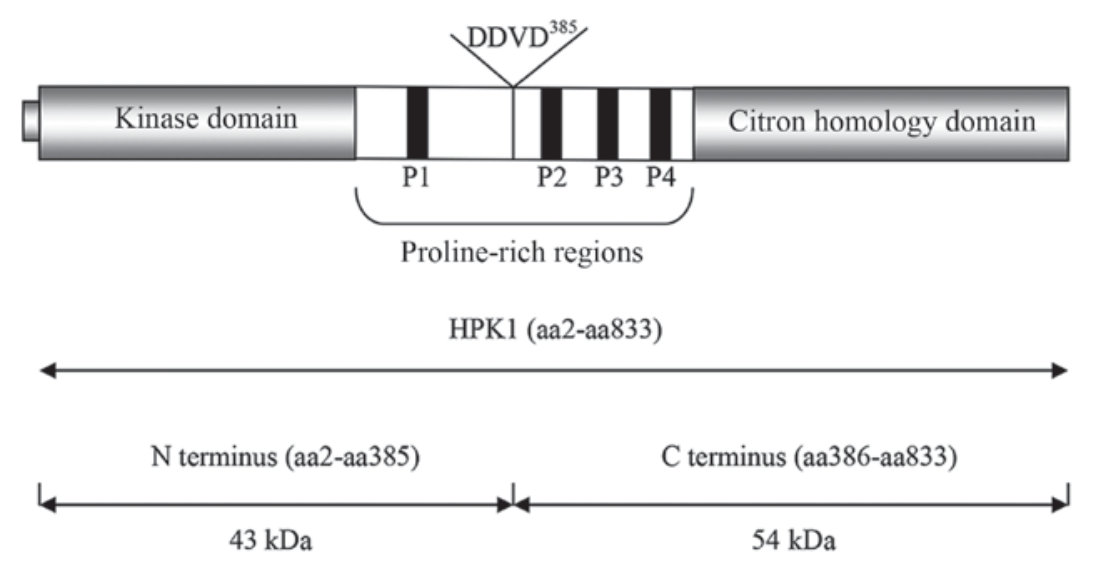

Figure 1. Schematic structure of HPK1. Full-length HPK1 is $97 \mathrm{kDa}$. It consists of an N-terminal kinase domain, four proline-rich regions (P1, P2, P3 and P4) and a citron homology domain at its C-terminus. HPK1 is cleaved into two fragments by caspase-3 during apoptosis at Asp385 (within a conserved DDVD 385 motif). The N-terminal region (43 kDa; aa2-aa385) contains the kinase domain and P1, whereas the C-terminal region (54 kDa; aa386-aa833), contains the citron homology domain and the other three proline-rich regions. HPK1, hematopoietic progenitor kinase 1.

\section{Adaptor proteins}

Adaptor proteins are defined as proteins, which exert no enzymatic activity (kinase or phosphatase) or transcriptional activity, but contain noncovalent protein-protein interaction domains, thus being able to selectively form multi-protein complexes and transmit upstream signals to downstream targets $(14,43,44)$. They usually localize close to the plasma membrane, and a number are directly associated with surface receptors or the cytoskeleton (18). Adaptor proteins are essential in relaying extracellular signals from the plasma membrane to the cell nucleus.

Those adaptor proteins, which interact with HPK1, usually contain SH2/SH3 domains. The SH2 domains can bind phosphorylated tyrosine residue, and the SH3 domains can bind the consensus sequence (PXXP), which is the minimal sequence requirement for $\mathrm{SH} 3$ domain ligands and is located within the proline-rich regions of HPK1 $(14,25,45)$. With multiple binding sites, these adaptor proteins can integrate and diversify cellular signals by linking different signaling proteins to create various combinations of multi-protein complexes $(45,46)$.

Upon receiving upstream signals, these $\mathrm{SH} / \mathrm{SH} 3$ adaptor proteins can recruit HPK1 from the cytoplasm to activated receptors, which have been tyrosine-phosphorylated, localized at the plasma membrane or glycolipid-enriched microdomains (GEMs or lipid rafts), which facilitates the subsequent activation of HPK1 and downstream signaling events $(13,14,47)$. Certain adaptor proteins can also link PTKs to HPK1 $(9,14)$.

This review aims to describe those adaptor proteins, which interact with HPK1, focusing particularly on their types of interaction with HPK1 and the effects of these interactions.

\section{CARD11}

Caspase recruitment domain family, member 11 (CARD11), also known as CARMA1 and Bimp3, belongs to the CARD family and to the membrane-associated guanylate kinase (MAGUK) family. The MAGUK family members usually contain between one and three PDZ domains (named after the domain-containing PSD-95, Dlg and ZO-1 proteins).
CARD11 contains one PDZ domain, followed by SH3 and GUK domains (48).

In addition to its C-terminal MAGUK-like features, CARD11 contains a CARD within its N-terminus. A coiled-coil domain is present after the CARD, and a linker region is present between the coiled-coil domain and PDZ domain (48).

It has been shown there is a constitutive association between HPK1 and CARD11 in T cells. The interaction between HPK1 and CARD11 is mainly mediated by the coiled-coil domain and CARD of CARD11; and HPK1 phosphorylates the linker region of CARD11 upon TCR stimulation (49). The activation of NF- $\kappa \mathrm{B}$ in response to antigen receptor stimulation is mediated by the IKK complex, and phosphorylation of the linker region of CARD11 is a precondition for IKK activation. Following phosphorylation, CARD11 interacts with B-cell lymphoma 10 and mucosa associated lymphoid tissue lymphoma translocation gene 1, and induces activation of the IKK complex. The loss of CARD11 causes disruption of IKK activation. Therefore, CARD11 is important for the activation of NF- $\kappa B$ via HPK1 in lymphocytes $(48,49)$. Ser551 in CARD11 is an important residue for the TCR-induced activation of NF- $\mathrm{kB}$ though HPK1, with Ser549 having an auxiliary role (49).

It has been reported that HPK1 and PKC $\theta$ alone can increase the CARD11-dependent activity of NF- $\mathrm{KB}$, and their combined expression is able to further augment the activation of NF- $\kappa \mathrm{B}$. This indicates that CARD11 can integrate HPK1-mediated and PKC $\theta$-mediated signaling events to enhance the activation of NF- $\mathrm{KB}(49)$.

\section{HS1}

Hematopoietic lineage cell-specific protein 1 (HS1) is mainly expressed in hematopoietic cells $(3,47,50)$. It contains several characteristic structures, including an $\mathrm{N}$-terminal actin-binding domain, proline-rich sequences and a C-terminal SH3 domain $(3,47)$. As an actin-binding protein, $\mathrm{HS} 1$ can bind to the actin cytoskeleton and orchestrate TCR or BCR signaling (51). It is reported to be involved in the activation of T/B cells and 
differentiation of erythroid cells. Upon hematopoietic cell antigen receptor engagement, it is tyrosine-phosphorylated by the Src family and Syk/ZAP-70 family $(3,18,50)$.

In hematopoietic cells, HS1 is constitutively associated with HPK1 through its SH3 domain $(3,18)$. However, the sites responsible for their binding in HPK1 remain to be elucidated. By synthesizing a series of peptide analogs derived from the proline-rich regions of HPK1 and examining their ability to bind the SH3 domain of HS1, it has been found that the typical class II PXXPXK binding motif is not sufficient to induce the interaction with the SH3 domain of HS1 $(3,52)$. An additive lysine, which locates two (in P2) or five (in P4) residues following the basal PXXPXK motif (PXXPXKXKX in P2 or PXXPXKXXXXK in P4) is required for the high-affinity binding of the HS1 SH3 domain (3). The finding that the cleaved HS1 SH3 domain and full-length HS1 exhibit similar affinities with the proline-rich regions of HPK1 demonstrates that the whole HS1 does not affect the affinity of its $\mathrm{SH} 3$ domain and the proline-rich regions of HPK1, and that the $\mathrm{SH} 3$ domain contains all binding recognition determinants (3).

The effects of the association between HS1 and HPK1 remain to be elucidated, however, it is suggested that this interaction forms a possible link between the HPK1-JNK pathway and HS1 signaling cascades (18).

\section{HIP-55}

HPK1-interacting protein of $55 \mathrm{kDa}$ (HIP-55), also termed DBNL, SH3P7 and mAbp1, is a novel member of the drebrin/Abp1 family of actin-binding proteins. The drebrin/Abpl family is conserved from yeast to mammals, and is characterized by the presence of a homologous N-terminal actin-depolymerizing factor homology domain $(50,53)$. This family includes SH3P7 in mice $(47,53)$ and Abp1 in yeast (53). The drebrin/Abp1 family, which binds filamentous actin but not actin monomers, is involved in multiple signaling events (53).

HIP-55 is a ubiquitously expressed protein, which consists of an actin-binding domain at its N-terminus, two consensus tyrosine phosphorylation sites, and an SH3 domain at its C-terminus $(50,53,54)$. Its C-terminal SH3 domain is homologous with HS1 (50).

HIP-55 has no actin polymerization activity (53). It binds to filamentous actin and colocalizes with actin filaments $(54,55)$. As an actin-binding protein, HIP-55 may affect immune synapse formation and TCR signaling by regulating cytoskeletal reorganization (54). HIP-55 is able to inducibly interact with and is phosphorylated by upstream ZAP-70 and Fyn following TCR stimulation (53-55). It is possible that HIP-55 may be involved in regulating the activity of ZAP-70 by modulating the recruitment of ZAP-70 to TCR via cytoskeletal reorganization in TCR signaling (54). It is also known that HIP-55 is involved in BCR signaling. As Syk shares similar regulatory mechanisms with ZAP-70, it is possible that HIP-55 may interact with Syk, and affect B cells or other antigen-presenting cells (APCs) via Syk (54).

In Jurkat T cells, HIP-55 constitutively binds HPK1, and can increase the kinase activity of HPK1 $(53,56)$. This interaction is mediated by the SH3 domain of HIP-55 and the P2 domain of HPK1 $(51,56)$.
Using 293T cells, the coexpression of HIP-55 with HPK1 has been shown to significantly increase HPK1 and JNK kinase activity $(55,56)$. The activation of JNK by HIP-55 is mediated by HPK1, as transfection with the kinase-dead HPK1 mutant (HPK1-K46M) completely inhibits the activation of JNK by HIP-55 (56). The knockdown of HIP-55 by RNA interference (RNAi) in Jurkat T cells or the knockout of HIP-55 in mouse $\mathrm{T}$ cells results in a marked decrease in the activity of HPK1 and JNK, but not that of extracellular signal-regulated kinase (ERK) kinase upon TCR stimulation $(54,55)$. Therefore, HIP-55 serves as an upstream activator of HPK1 and the JNK signaling pathway (56).

HIP-55 also cooperates with HPK1 in inhibiting the activity of nuclear factor of activated T cell (NFAT) in T cells. Overexpressing HIP-55 or HPK1 alone leads to inhibition of the transcriptional activity of NFAT following TCR crosslinking, and this inhibitory effect is further augmented by the coexpression of two proteins. Transfection with the kinase-dead HPK1 mutant (HPK1-K46E), or knockdown of either HIP-55 or HPK1 by RNAi, leads to the activity of NFAT increasing in these systems $(10,53)$.

Upon TCR/BCR stimulation, HIP-55 and HPK1 translocate to the T/B cell-APC junction and GEMs (53-55). The SH3 domain and actin-binding domain of HIP-55 are essential for its locating in the T/B cell-APC interface (53). It has been suggested that HIP-55 may bind HPK1 and mediate the recruitment of HPK1 to the T/B cell-APC interface, where HPK1 interacts with its substrates and downregulates the TCR/BCR signaling pathway $(10,47,53)$.

\section{GRB2 family}

The growth factor receptor-bound protein 2 (GRB2) family consists of growth factor GRB2, GRB2-related adaptor protein (GRAP) and GRB2-related adaptor downstream of Shc (GADS), also termed GRAP2, GRAPL, Grf40, Grid and Mona $(5,43,45)$. The GRB2 family contains one $\mathrm{N}$ - and one C-terminal SH3 domain flanking a central SH2 domain; and GADS possesses a 120-amino acid unique region, which is rich in glutamine and proline residues and is between the $\mathrm{SH} 2$ domain and C-terminal SH3 domain $(5,45)$. A database search indicated that this unique region has no apparent homology with other proteins (45). GRAP has a similar $\mathrm{SH} 2$ and $\mathrm{SH} 3$ binding profile to GRB2 $(5,57)$. GADS shares $40-50 \%$ sequence homology in the SH3 domains and 57\% homology in the SH2 domain with GRB2 and GRAP (45), however, the SH3 domains of GADS cannot interact with certain proline-rich proteins $(5,43)$. GRB2 is expressed ubiquitously, whereas the expression of GRAP and GADS are restricted to hemopoietic cells $(5,45)$.

GRB2 and GRAP constitutively associate with HPK1 $(9,13,57)$. The N-terminal SH3 domain of GRB2 binds to the consensus motif (PXXPXR/K) $(14,45,58)$. It has been shown that P1, P2 and P4 of HPK1 bind to the N-terminal SH3 domain of GRB2, and P2 is the primary site for GRB2 binding to HPK1, whereas P4 is the last of the three $(13,14,58)$. However, the exact binding domain of HPK1 to GRAP remains to be elucidated.

GADS and HPK1 can form a complex in cells, and the TCR-induced phosphorylation of HPK1 is able to increase its 
ability to bind GADS $(5,25,45)$. The C-terminal SH3 domain of GADS mediates the binding with P2 (45) or P4 (5) of HPK1, and the SH2 domain is associated with the tyrosine phosphorylation of HPK1 (5).

Previous X-ray crystallography studies have found that the typical $\beta$-barrel SH3 domain in GADS presents the binding pocket to HPK1; there are 16 structured residues in HPK1 composing a peptide, which mediates the HPK1-GADS interaction. The specific structural characteristics of the peptide comprise a $3_{10}$ helix between residues Arg9 and Lys12, and a polyproline type II (PPII) helix between residues Gln2 and Pro7. The PXVPXRXXK residues of this peptide are essential for mouse HPK1 binding to the GADS C-terminal SH3 domain, and the residues RXXK, which form the $3_{10}$ helix, are the core motif. However, in the human HPK1 sequence, the arginine can be substituted with a lysine, leaving only the last lysine residue as a strictly conserved position. A PXXP motif, which belongs to the PPII helix, is also essential for the interaction of GADS and HPK1 (59).

In $\mathrm{T}$ cells, the $\mathrm{SH} 2$ and $\mathrm{SH} 3$ domains of GRB2 and GRAP bind to similar proteins (5); in B cells, the absence of GRB2 or GRAP only mildly reduces the kinase activity of HPK1 upon BCR stimulation, whereas the absence of both in B cells severely impairs HPK1 kinase activity upon BCR stimulation $(9,57)$. GRAP null mice exhibit no apparent phenotypes (5). These findings suggest that GRB2 and GRAP may share overlapping functions.

GRAP is able to inhibit the ERK pathway without affecting the activation of JNK (60), which may provide an explanation for how HPK1 negatively affects the ERK pathway in T cells.

In $\mathrm{T}$ cells, it has been suggested that GRB2 and GRAP couple PTKs to the Ras pathway through the recruitment of GRB2-son of sevenless homolog (SOS) or the GRAP-SOS complex to tyrosine-phosphorylated linker for activation of T cells (LAT) $(5,57)$. By contrast, GRB2 has been shown to couple upstream PTKs, including Src, to HPK1 $(13,18)$. As GRB2 can couple PTKs to the Ras pathway and to HPK1, it may serve as a molecular bridge between these two biochemical pathways and lead to interpathway cross-talk between these signaling pathways (13).

GRB2 couples HPK1 not only to the TCR signaling pathway, but also to the EGF receptor signaling pathway. Upon EGF stimulation, GRB2 mediates the recruitment of HPK1 to the autophosphorylated EGF receptor tail and to the Shc docking protein, and HPK1 is phosphorylated on tyrosine $(9,13)$.

The cotransfection of GADS and HPK1 increases the kinase activity of HPK1, suggesting that GADS may recruit HPK1 to the proximity of other HPK1 activators; the coexpression of GADS and HPK1 also increases HPK-mediated activation of the JNK pathway, and the additive effect is mediated by the specific GADS-HPK1 interaction; in Jurkat T cells, the cotransfection of HPK1 and GADS also has an additive effect by increasing the transcriptional activation of IL-2 following TCR stimulation (45). It may be that, upon TCR stimulation, GADS couples TCR and CD28 with PTKs to HPK1, facilitating the phosphorylation and activation of HPK1 by PTKs, and transmitting the signals downstream to result in activation of the JNK pathway and the transcription of IL-2 (45).
GADS facilitates the phosphorylation of HPK1, however, HPK1 also phosphorylates Thr254 (mouse) or Thr262 (human) of GADS, which mediates the binding of 14-3-3\} to GADS (61). GADS can also inducibly associate with LAT in T cells, but it does not form a complex with SOS. Instead, GADS binds to the $\mathrm{SH} 2$ domain-containing leukocyte protein of $76 \mathrm{kDa}$ (SLP-76)/B-cell linker (BLNK), which is important in transmitting the signaling of TCR/BCR. Therefore, through its association with SLP-76/BLNK and LAT, GADS is central in transmitting the signaling from the activated $\mathrm{TCR} / \mathrm{BCR}(5,62)$.

The three members of the GRB2 family are known to be involved in forming distinct signaling complexes during TCR stimulation $(5,14)$, indicating that these adaptors are involved in the TCR signaling pathway. Although the majority of signaling molecules involved in TCR signaling have their counterparts in B cells, no functional analog of LAT has been identified in B cells (62), therefore, the mechanisms mediating the recruitment of the GRB2 family to BCR remain to be elucidated.

\section{LAT}

LAT is a $36-38 \mathrm{kDa}$ palmitoylated transmembrane-associated adaptor protein. It is localized in GEMs $(9,54,55)$. Following TCR stimulation, LAT can be heavily tyrosine phosphorylated by the Syk/ZAP-70 family $(9,47)$. It subsequently serves as a docking protein for several $\mathrm{SH} 2$ domain-containing signaling molecules, including SLP-76, GRB2, GADS, phospholipase C (PLC) $-\gamma 1$, and the p85 subunit of phosphatidylinositol 3-kinase (PI3K) $(9,54,55)$. The LAT-associated proteins recruit various signaling molecules to GEMs, where these signaling molecules are activated and promote multiple downstream signaling events, including the activation of MAPK, release of intracellular calcium and increased production of IL-2 $(54,55)$.

It has been shown that the presence of LAT is required for TCR-induced HPK1 activation (4). In Jurkat T cells, LAT can form an inducible complex with HPK1 as early as 1 min post-TCR stimulation, and LAT recruits HPK1 into GEMs, leading to the activation of HPK1 by PTKs $(6,9)$. The proline-rich regions of HPK1 are critical to the HPK1-LAT interaction. As LAT does not contain an SH3 domain for its direct binding to the HPK1 proline-rich regions, the inducible HPK1-LAT complex formation is likely via $\mathrm{SH} 2 / \mathrm{SH} 3$ adaptors. It is possible that GRB2 or GADS mediate the HPK1-LAT interaction, which bind LAT through the SH2 domain and interact with HPK1 by the SH3 domain. GRB2 or GADS may bring HPK1 to the LAT signaling complex, where HPK1 interacts with its effector molecules and substrates upon TCR stimulation $(9,47)$.

\section{SLP-76 family}

The SLP-76 family includes SLP-76, its B-cell homolog BLNK (also termed SLP-65 or BASH), and cytokine-dependent hematopoietic cell linker (CLNK; also termed MIST) $(57,62,63)$. They all contain a similar structure with an $\mathrm{N}$-terminal acidic region, which contains a tyrosine-based $\mathrm{SH} 2$ domain-binding motif, a central proline-rich region, which interacts with SH3 
domains, and a C-terminal SH2 domain, which interacts with phosphotyrosine (46,63-65).

SLP-76 is widely expressed in T cells, natural killer (NK) cells, myeloid cells, platelets and mast cells; BLNK accumulates predominantly in B cells, whereas, CLNK is contained exclusively in cytokine-stimulated hemopoietic cells, including IL-2-induced T cells and NK cells, and IL-3-induced myeloid cells and mast cells (64).

SLP-76 and BLNK are adaptor proteins specific to T cells and $\mathrm{B}$ cells, respectively, and they are crucial in the signal transmission of TCR and BCR $(59,63,66)$. Upon TCR or BCR stimulation, SLP-76 and BLNK are tyrosine phosphorylated by the Syk/ZAP-70 family members, and translocate into and recruit several signaling molecules into GEMs, where the activated TCR and PTKs can activate downstream effectors $(6,62,63)$. They can interact with several components of TCR or BCR signaling pathways, including Vav, non-catalytic region of tyrosine kinase adaptor protein (NCK), GADS, GRB2, Btk, Itk, Cb1, PLC- $\gamma 1$ and PLC- $\gamma 2$ (47,62,63). They also bind HPK1 following TCR or BCR stimulation $(6,32,47)$. It has been reported that GRB2, GADS and LAT interact with SLP-76/BLNK, and may mediate the interactions between SLP-76/BLNK and HPK1 $(62,63,67)$.

The majority of HPK1-adaptor proteins interactions are mediated by the proline-rich regions of HPK1 and the SH3 domains of adaptor proteins; however, the HPK1-SLP-76 and HPK-BLNK interactions are mediated by Tyr379 (mouse) or Tyr381 (human), located in the N-terminal of HPK1 P2, and phosphorylated by the Syk/ZAP-70 family following TCR or BCR stimulation and the SH2 domains of SLP-76 and BLNK $(10,62,63,65)$.

When T cells lack SLP-76 or B cells lack BLNK, the kinase activity of HPK1 induced by TCR or BCR stimulation is reduced $(57,62,63)$. It is noted that BLNK contributes to the activation, but not to the tyrosine-phosphorylation, of HPK1 by BCR stimulation (63).

SLP-76 is involved in activating HPK1, however, HPK1 also phosphorylates the serine and threonine residues of SLP-76 $(6,51,67)$. It has been shown that HPK1 is able to directly phosphorylate the serine residues of SLP-76, consequently creating 14-3-3-binding sites, the critical one being phosphorylated Ser376, on SLP-76 and inducing the binding of 14-3-3६ (67) and $\tau$ (6) isoforms to serine-phosphorylated SLP-76. The protein $14-3-3 \xi$ and $\tau$ are negative regulators of the TCR signaling pathway $(6,67)$. They can bind and inhibit PI3K and PKC $\theta$. PI3K can trigger TCR-induced calcium signaling, and $\mathrm{PKC} \theta$ can promote TCR-induced ERK phosphorylation (6). PKC $\theta$ is also able to increase the activity of RAP1 (51). RAP1 can mediate LFA-1 integrin activation through its binding to RAP ligand, a regulator for cell adhesion and polarization enriched in lymphoid tissues, which assists $\mathrm{T}$ cells in adhering to ICAM-1 and spreading (51). Upon TCR stimulation, adhesion and degranulation promoting adaptor protein (ADAP) binds SLP-76, and their binding causes membrane recruitment of active RAP1. HPK1, which also binds SLP-76, competes with ADAP for SLP-76 and reduces the recruitment of RAP1 (51). The phosphorylation of Ser376 also mediates SLP-76 proteasomal degradation and Lys30 ubiquitination. The Lys-30 ubiquitination of SLP-76 can attenuate the activation of JNK and ERK (66). Lasserre et al (61) reported that, following activation, HPK1 is incorporated into SLP-76-containing microclusters, subsequently phosphorylating SLP-76 on Ser376 and GADS on Thr254 (mouse) or Thr262 (human), respectively. These modifications recruit the 14-3-3 $\zeta$ protein dimer, which in turn dissociates the SLP-76-GADS-14-3-3 $\zeta$ complex from phosphorylated LAT and prevents its incorporation into a new one, consequently reducing the persistence of SLP-76-containing microclusters and downregulating TCR signaling (61). These results explain, at least in part, the mechanisms through which HPK1 causes downregulation of the TCR signaling pathway and inhibition of $\mathrm{T}$ cell activity.

The TCR-induced phosphorylation of SLP-76 tyrosine is higher in $\mathrm{HPK} 1^{-/-} \mathrm{T}$ cells and thymocytes than in wild-type cells, and it has been found that several serine and threonine residues are located in the proximity of three primary phosphotyrosine sites (Tyr113, Tyr128 and Tyr145) on SLP-76. Therefore, it is possible that the phosphorylation of SLP-76 serine and threonine residues mediated by HPK1 changes the conformation of SLP-76, and results in altered protein-protein interactions and decreased tyrosine phosphorylation, creating further effects (6). Sauer et al (62) reported that HPK1 can inhibit the activation of activator protein-1 (AP-1), and this depends on it antagonizing the recruitment of positive effectors into the SLP-76 SH2 domain.

Following BCR stimulation, HPK1 is also able to phosphorylate BLNK Thr152, which mediates BLNK/14-3-3 binding. Thr152 phosphorylation also induces BLNK proteasomal degradation and ubiquitination at Lys37, Lys38 and Lys42. It has been found that an E3 ligase is recruited to BLNK via 14-3-3, mediating and inducing BLNK ubiquitination. These ubiquitination sites can inhibit the activation of ERK, JNK and IKK. All of these, in turn, attenuate BCR signaling and B cell activation (32).

Unlike SLP-76, HPK1 can induce BLNK to interact with all seven isoforms of 14-3-3, with the relative affinity order $\gamma>$ $\tau>\eta>\varepsilon>\beta>\delta>\zeta$. In contrast to HPK1-deficient T cells, in which the SLP-76/14-3-3 interaction is only partially lost, BLNK/14-3-3 binding is undetectable in HPK1-deficient B cells, suggesting HPK1 is involved exclusively in B cells (32).

As mentioned previously, HPK1 can activate JNK (16,17,20-22) and IKK (23). However, through SLP-76/BLNK, HPK1 also has an inhibitory effect on the activation of JNK and IKK. In an HPK1-overexpression system, the expression of SLP-76/BLNK is limited for HPK1-mediated feedback inhibition. Consequently, the positive effect of HPK1 on the activation of JNK and IKK can overcome the inhibitory effect. However, in HPK1-deficient cells, the inhibitory role of HPK1 is predominant $(32,66)$.

CLNK is the third member of the SLP-76 family. It is a cytokine inducible (IL-2 and IL-3) adaptor protein (47). Unlike SLP-76 and BLNK, CLNK does not interact with Vav, NCK, GRB2, GADS or PLC- $\gamma$ (64), and it is not required for immune system functions (68).

Upon TCR engagement, CLNK binds HPK1 with its SH2 domain. The conserved residue, Arg335, within the CLNK SH2 domain, and phosphorylated Tyr379 (mouse) or Tyr381 (human) within HPK1 is essential for their interaction $(10,64)$. However, deletion of the CLNK SH2 domain only partially $(\sim 75 \%)$ reduces the ability of CLNK to bind with HPK1 in 
Cos- 1 cells. Therefore, there may be additional motifs involved in the CLNK-HPK1 binding (64). The association between CLNK-HPK1 is significantly higher, compared with that of SLP-76-HPK1 $(10,64)$. The expression of CLNK is able to augment the extent of tyrosine phosphorylation and kinase activity of HPK1, and CLNK is also phosphorylated by HPK1 $(10,64)$.

The coexpression of CLNK and Lck can result in HPK1 membrane recruitment, which in lymphocytes results in the recruitment of HPK1 to the contact site of T-cell-APC conjugates, where HPK1 is tyrosine phosphorylated and activated. However, CLNK alone is incapable of localizing HPK1 to the plasma membrane; HPK1 appears to divert CLNK away from the plasma membrane (10).

It has been reported that the expression of HPK1 alone is able to inhibit the activity of the IL-2 promoter, and the introduction of CLNK alone can increase the activation of the IL-2 promoter, although the coexpression of CLNK and HPK1 causes more pronounced activation of the IL-2 promoter, compared with CLNK alone. Their cooperation requires the enzymatic activity of HPK1, as the kinase-inactive HPK1 (K46E) inhibits the ability of CLNK to upregulate IL-2 promoter activity (64).

It has been suggested that CLNK may permit the involvement of HPK1 in a signaling pathway, which differs from that used by HPK1 alone. In this signaling pathway, HPK1 turns from an inhibitor to a positive regulator of IL-2 promoter activation. This change may be due to a CLNK-mediated change in HPK1 cellular localization or a modification in its substrate specificity (64).

CLNK and SLP-76 may transmit immunoreceptor signaling in a similar manner but using different sets of partners. CLNK can restore antigen receptor-induced transcriptional events in a manner analogous to that of SLP-76 in SLP-76-deficient cells, indicating that CLNK is capable as a functional substitute for SLP-76 in immunoreceptor signaling (64).

Through CLNK, certain requirements for immunoreceptor signaling, including the formation of a proper SLP-76-GADS-LAT complex in T cells, may be alleviated, resulting in the facilitation of cell activation. In $\mathrm{T}$ cells, this property may reduce the requirements for engagement of coreceptors (CD4 and CD8) and costimulatory molecules (CD28), which has been described in memory T cells (64). Therefore, the interaction between CLNK and HPK1 indicates the possibility that HPK1 can be involved in T cells which have been activated by cytokines, for example memory T cells (47).

\section{CRK family}

V-crk avian sarcoma virus CT10 oncogene homolog (CRK) adaptor proteins consist of CRK I-, CRK II- and CRK-like protein (CRKL) $(14,69)$. All CRK proteins contain SH2 and SH3 domains $(58,69)$. CRKI and CRKII result from alternative RNA splicing. CRK I consists of one N-terminal SH2 domain followed by one SH3 domain, and CRK II consists of one N-terminal SH2 domain followed by two tandem SH3 domains; CRKL, which is cloned from chronic myelogenous leukemia cells, shares $60 \%$ overall homology of CRK II and contains two SH3 domains $(14,43)$. CRK I is expressed in embryonic cells, whereas CRK II and CRKL are widely expressed (43).

In Jurkat $\mathrm{T}$ cells, CRK inducibly binds HPK1 following TCR stimulation for $10 \mathrm{~min}$, with CRKL forming a constitutive complex with HPK1 (9). The P2 and P4 of HPK1, which contain the consensus motif (PXLPXK), may be involved in recognition of the CRK family $(14,70,71)$. This consensus motif is unique and incompatible with the majority of other $\mathrm{SH} 3$ domain-binding motifs (58). HPK1 P2, the sequence of which is fully conserved between mouse and humans, is considered to be the primary site for the CRK family binding. The Lys 400 of P2 is important in the recognition of CRK and CRKL by HPK1 (14). In terms of HPK1 P4, its sequence is not conserved between humans and mouse; therefore, it is not likely to be essential for the interaction with CRK/CRKL $(14,58)$. The members of the CRK family preferentially use their first SH3 domain to bind HPK1 $(9,14,58)$, and the binding is independent of the catalytically activity of HPK1, as the inactive HPK1 can form more stable binding with the CRK proteins than active HPK1 (58).

HPK1 can phosphorylate CRK mainly on threonine, with weaker phosphorylation on serine, whereas HPK1 phosphorylates CRKL mainly on serine with weaker phosphorylation on threonine $(14,47)$.

Upon TCR stimulation, CRK and CRKL are shown to form inducible complexes with tyrosine kinases ZAP-70 and Fyn, respectively (9). Therefore, CRK and CRKL may couple HPK1 to ZAP-70 and Fyn, respectively, to become involved in the TCR-mediated signaling events following TCR engagement. In addition to TCR stimulation, HPK1 is tyrosine-phosphorylated by EGF stimulation and is recruited to the EGF receptor tail via the CRK adaptors, suggesting that the CRK adaptors are also involved in the recruitment of HPK1 to the EGF receptor $(9,14)$.

In 293 T cells, the CRK family can activate HPK1, and shares common downstream effectors, including TAK1, MEKK1 and MKK4, for the activation of JNK with HPK1; the CRK family can cooperate with HPK1 to activate JNK synergistically when they are coexpressed. These data indicate that the CRK proteins function as upstream regulators of the HPK1-mediated JNK signaling cascade (14). In contrast to the results in 293T cells, the overexpression of CRKL and HPK1 does not further activate JNK in Cos-7 cells (58). These results indicate the role of CRKL in modulating the activation of HPK1 and activity of JNK depends on the cell types or the accessory proteins of every cell type (14). Upon stimulation, the CRKL-HPK1 interaction may also translocate HPK1 from the cytoplasm to areas of signal transduction, including GEMs (47).

CRK interacts with two guanine nucleotide exchange factors for Ras family members, SOS and RAP guanine nucleotide exchange factor 1 (RAPGEF1), also known as C3G. SOS is a known activator of Ras, and RAPGEF1 is an exchange factor of RAP1A and RAP1B. CRK may be involved in the activation of JNK through RAPGEF1 $(14,43)$. CRKL has also been shown to activate the Ras and JNK signaling pathways in cells (14). HPK1 has the potential to inhibit RAP1 by sequestration of RAPGEF1 though the interaction of HPK 1 and CRKL, leading to the inhibition of $\mathrm{T}$ cell activity (51). 
Table I. Adaptor proteins binding HPK1.

\begin{tabular}{|c|c|c|c|c|}
\hline Adaptor protein & Structure & Mode of binding & $\begin{array}{l}\text { Binding domain } \\
\text { within HPK1 }\end{array}$ & $\begin{array}{l}\text { Binding domain } \\
\text { within adaptor }\end{array}$ \\
\hline CARD11 & CARD-(coiled-coil)-linker-PDZ-SH3-GUK & Constitutive & Undetermined & CARD, coiled-coil \\
\hline HS1 & $\mathrm{AB}^{\mathrm{a}}-\mathrm{PR}^{\mathrm{b}}-\mathrm{SH} 3$ & Constitutive & Undetermined & SH3 \\
\hline HIP-55 & (ADF-H)-pTyr-SH3 & Constitutive & $\mathrm{P} 2$ & SH3 \\
\hline GRB2 & SH3-SH2-SH3 & Constitutive & $\mathrm{P} 1, \mathrm{p} 2, \mathrm{p} 4$ & $\mathrm{SH} 3\left(\mathrm{~N}^{\mathrm{c}}\right)$ \\
\hline GRAP & SH3-SH2-SH3 & Constitutive & Undetermined & Undetermined \\
\hline GADS & SH3-SH2-120aad-SH3 & Inducible & $\mathrm{P} 2, \mathrm{p} 4$ & $\mathrm{SH} 3\left(\mathrm{C}^{\mathrm{e}}\right)$ \\
\hline LAT & $\mathrm{EX}^{\mathrm{f}}-\mathrm{TM}^{\mathrm{g}}-\mathrm{pTyr}$ & Inducible & Indirect & Indirect \\
\hline SLP-76 & pTyr-PR-SH2 & Inducible & $\begin{array}{l}\operatorname{Ptyr} 379\left(\mathrm{~m}^{\mathrm{h}}\right), \\
\operatorname{Ptyr} 381\left(\mathrm{~h}^{\mathrm{i}}\right)\end{array}$ & SH2 \\
\hline BLNK & pTyr-PR-SH2 & Inducible & $\begin{array}{l}\text { Ptyr379(m), } \\
\text { Ptyr381(h) }\end{array}$ & $\mathrm{SH} 2$ \\
\hline CLNK & pTyr-PR-SH2 & Inducible & $\begin{array}{l}\text { Ptyr379(m), } \\
\text { Ptyr381(h) }\end{array}$ & $\mathrm{SH} 2$ \\
\hline CRK I & $\mathrm{SH} 2-\mathrm{SH} 3$ & Inducible & $\mathrm{P} 2, \mathrm{p} 4$ & SH3 \\
\hline CRK II & SH2-SH3-SH3 & Inducible & $\mathrm{P} 2, \mathrm{p} 4$ & SH3(N) \\
\hline CRKL & SH2-SH3-SH3 & Constitutive & $\mathrm{P} 2, \mathrm{p} 4$ & $\mathrm{SH} 3(\mathrm{~N})$ \\
\hline BAM32 & SH2-PH & Constitutive & Undetermined & Undetermined \\
\hline NCK & SH3-SH3-SH3-SH2 & Inducible & All SH3 & All PR \\
\hline
\end{tabular}

${ }^{\mathrm{a}}$ Actin-binding domain; ${ }^{\mathrm{b}}$ proline-rich region; ${ }^{\mathrm{C}} \mathrm{N}$-terminal; ${ }^{\mathrm{d}}$ amino acid; ${ }^{\mathrm{e}} \mathrm{C}$-terminal; ${ }^{\mathrm{f}}$ extracellular domain; ${ }^{\mathrm{g}}$ transmembrane domain; ${ }^{\mathrm{h}} \mathrm{mouse}$; ihuman. HPK1, hematopoietic progenitor kinase 1.

\section{BAM32}

B-cell adaptor molecule of $32 \mathrm{kDa}$ (BAM32), also known as DAPP1 and PHISH, is a recently identified adaptor protein, which is restricted primarily to hematopoietic cells with particularly high expression in B cells (72). BAM32 consists of an N-terminal SH2 domain and a C-terminal pleckstrin homology $(\mathrm{PH})$ domain. It is recruited to the plasma membrane in a PI3K-dependent manner following BCR crosslinking, and appears to function as a dual adaptor for phosphotyrosine and 3-phosphoinosides (46). BAM $32^{-/-} \mathrm{B}$ cells are defective in $\mathrm{T}$ cell-independent, but normal in $\mathrm{T}$ cell-dependent responses to antigens in vivo (46).

BAM32 associates with HPK1, and this is not dependent on BCR stimulation (46); it can directly localize fHPK1 to the plasma membrane (46). BAM32 is important for the activation of HPK1, ERK and JNK following BCR stimulation. BAM32 ${ }^{-/-}$B cells show defects in the activities of HPK1, ERK and JNK in response to BCR crosslinking, however, p38 is not affected in the absence of BAM32 $(47,50)$. The exact association between BAM32 and HPK1 requires further investigation.

\section{NCK}

NCK includes $\mathrm{NCK} \alpha$ and $\mathrm{NCK} \beta, \mathrm{NCK} \alpha$ is encoded by the nck gene, whereas NCK $\beta$, also known GRB4, is encoded by a novel gene. NCK $\beta$ shares $68 \%$ amino acid identity with $\mathrm{NCK} \alpha$ (43). The NCK proteins contain one $\mathrm{SH} 2$ domain and three $\mathrm{SH} 3$ domains, and can be phosphorylated on serine, threonine and tyrosine residues (47).
NCK $\alpha$ can interact directly with HPK1 $(9,13)$, although the interaction of NCK $\beta$ with HPK1 remains to be elucidated. Using the yeast two-hybrid assay, the three NCK SH3 domains are able to interact with any of the four proline-rich motifs of HPK1, indicating that NCK may recognize multiple HPK1 proline-rich regions (13). It has been shown that the binding between NCK and HPK1 occurs primarily through the second $\mathrm{SH} 3$ domain of NCK, which is also known to bind the SOS and Abl proteins (58).

In Jurkat T cells, the interaction between HPK1 and NCK occurs following TCR stimulation for $1 \mathrm{~min}$, and the HPK1-NCK complex is sustained until $10 \mathrm{~min}$ of TCR stimulation. The kinetics of the interaction between HPK1 and NCK correlates with that of the activation of HPK1 during TCR stimulation, suggesting that NCK may be involved in the regulation of HPK1 during TCR stimulation (9).

Upon TCR stimulation, NCK and HPK1 translocate to GEMs $(9,53)$, where NCK can interact with the CD3 $\varepsilon$ chain and modulate downstream signals (44). It has been suggested that NCK may be involved in recruiting HPK1 to the activated TCR complex and GEMs, and transmitting upstream signals (47).

The interaction between HPK1 and NCK is induced not only by TCR stimulation, but also by EGF stimulation (13). The overexpression of GRB2 can significantly decrease the association between HPK1 and NCK, suggesting that these two adaptors can compete with each other for binding HPK1 (13). NCK also interacts with the SH2 domain binding motifs in the N-terminal region of SLP-76, which encompass phosphorylated Tyr113, Tyr128 and Tyr145 $(61,67)$. 
Table II. Functions of adaptor proteins.

\begin{tabular}{|c|c|c|c|}
\hline Adaptor & Other name & Family & Function \\
\hline CARD11 & $\begin{array}{l}\text { CARMA1, } \\
\text { Bimp3 }\end{array}$ & CARD/MAGUK & Activates IKK and NF-кB \\
\hline HS1 & - & - & Affects HPK1-JNK pathway \\
\hline HIP-55 & $\begin{array}{l}\text { DBNL, SH3P7, } \\
\text { mAbp1 }\end{array}$ & drebrin/Abp1 & $\begin{array}{l}\text { Activates HPK } 1 \text { and JNK; } \\
\text { inhibits NFAT and TCR/BCR signaling }\end{array}$ \\
\hline GRB2 & - & GRB2 & $\begin{array}{l}\text { Activates HPK1; affects Ras/EGFR/ } \\
\text { TCR/BCR signaling }\end{array}$ \\
\hline GRAP & - & GRB2 & $\begin{array}{l}\text { Activates HPK1; inhibits ERK; affects } \\
\text { Ras/TCR/BCR signaling }\end{array}$ \\
\hline GADS & $\begin{array}{l}\text { GRAP2, GRAPL, Grf40, } \\
\text { GRID, Mona }\end{array}$ & GRB2 & $\begin{array}{l}\text { Activates HPK1, JNK and IL-2; } \\
\text { affects TCR/BCR signaling }\end{array}$ \\
\hline LAT & - & - & $\begin{array}{l}\text { Activates HPK1; composes } \\
\text { platform for signal complexes }\end{array}$ \\
\hline SLP-76 & - & SLP-76 & $\begin{array}{l}\text { Activates HPK1; inhibits JNK, ERK; } \\
\text { AP-1 and TCR signaling; composes } \\
\text { platform for signal complexes }\end{array}$ \\
\hline BLNK & SLP-65, BASH & SLP-76 & $\begin{array}{l}\text { Activates HPK1; inhibits JNK, ERK, } \\
\text { IKK and BCR signaling; composes } \\
\text { platform for signal complexes }\end{array}$ \\
\hline CLNK & MIST & SLP-76 & $\begin{array}{l}\text { Activates HPK } 1 \text { and IL-2; functionally } \\
\text { substitutes for SLP-76 }\end{array}$ \\
\hline CRK I & - & CRK & Activates HPK1 and JNK \\
\hline CRK II & - & CRK & Activates HPK1 and JNK \\
\hline CRKL & - & CRK & Activates HPK1 and JNK \\
\hline BAM32 & DAPP1, PHISH & - & Activates HPK1, JNK and ERK \\
\hline NCK & - & - & Activates HPK1; affects TCR signaling \\
\hline
\end{tabular}

\section{Conclusions and future directions}

The features of the adaptor proteins described above are summarized in Tables I and II. As mentioned above, these adaptor proteins do not interact with HPK1 separately, they also associate with each other. Among these adaptors, LAT and SLP-76/BLNK have a specific status; they serve as docking proteins to form a platform for signal complexes. Following TCR/BCR stimulation, LAT and SLP-76/BLNK are tyrosine phosphorylated by the Syk/ZAP-70 family. SLP-76/BLNK is recruited onto LAT via their association with the GRB2 family $(61,62,67)$ to form a central scaffold, and their phosphotyrosines provide docking sites for $\mathrm{SH} 2$ and phosphotyrosine-binding domains of enzymes and other adaptor proteins $(10,62)$. This promotes the activation of effector molecules, including HPK1.

There are several controversies surrounding the data on HPK1. For example, it has been shown that the kinase-dead mutant of HPK1 cannot inhibit the activation of JNK by anti-CD3 and anti-CD28 co-stimulation (9,57); another study reported that the kinase-dead mutant of HPK1 inhibits the Vav-mediated activation of JNK following TCR stimulation (73). Kiefer et al (70) reported that HPK1 does not affect the ERK family following transfection into Cos-1 cells, whereas others have reported that HPK1 negatively affects the ERK signaling pathway in an overexpression system using $\mathrm{T}$ cells $(57,62)$, and that HPK $1^{-1-} \mathrm{T}$ cells and thymocytes enhance the TCR-induced activation of ERK (6). Reports have indicated that HPK1 negatively affects the transcriptional activity of AP-1 $(6,57,62,64)$, whereas others have shown that HPK1 enhances the transcriptional activity of AP-1 $(14,16,39,45,71)$. Previous studies have indicated that HPK1 is a negative regulator of TCR-induced IL-2 gene transcription $(4,6,11,64,67)$, whereas others have reported that HPK1 can enhance the transcriptional activity of IL-2 $(14,24,45,49,71)$.

In terms of cellular proliferation, there is data showing that HPK1 can assist $\mathrm{PGE}_{2}$ in suppressing $\mathrm{T}$ cell proliferation (11), and that $\mathrm{HPK} 1^{-/-} \mathrm{T}$ cells are hyperproliferative in response to TCR stimulation (6). Nagata et al (18) confirmed that antisense S-oligonuleotides to HPK1 mRNA can significantly inhibit EPO-dependent FD-EPO cell growth in a dose-dependent manner.

It has been reported that the overexpression of HPK1 promotes spontaneous apoptosis and anti-CD3-mediated apoptosis of T cells (26); HPK $1^{-/} \mathrm{T}$ cells have been shown to withstand $\mathrm{PGE}_{2}$-mediated $\mathrm{T}$ cell apoptosis (11), however, Shui et al (6) reported that $\mathrm{HPK} 1^{-/} \mathrm{T}$ cells undergo apoptosis in a similar manner to wild-type $\mathrm{T}$ cells following stimulation with anti-CD3 or anti-Fas. Others have shown that the siRNA-mediated knockdown of HPK1 increases T cell apoptosis induced by TCR stimulation (7). 
The conflicting findings reported may result from using different cell types and stimuli. Which factors have definitive roles in these events remain to be elucidated. It may be that these adaptor proteins couple HPK1 with various effector molecules, which leads HPK1 to different signaling pathways, and/or alters the cellular localization of HPK1, or they may modify substrate specificity to determine the functions of HPK1. This requires further investigation.

\section{Acknowledgements}

This study was supported by the National Natural Science Foundation of China (grant no. 81301359).

\section{References}

1. Alzabin S, Bhardwaj N, Kiefer F, Sawasdikosol S and Burakoff S: Hematopoietic progenitor kinase 1 is a negative regulator of dendritic cell activation. J Immunol 182: 6187-6194, 2009.

2. Zhou G, Boomer JS and Tan TH: Protein phosphatase 4 is a positive regulator of hematopoietic progenitor kinase 1 . J Biol Chem 279: 49551-49561, 2004

3. Siligardi G, Ruzza P, Hussain R, Cesaro L, Brunati AM, Pinna LA and Donella-Deana A: The SH3 domain of HS1 protein recognizes lysine-rich polyproline motifs. Amino Acids 42: $1361-1370,2012$

4. Sawasdikosol S, Pyarajan S, Alzabin S, Matejovic G and Burakoff SJ: Prostaglandin E2 activates HPK1 kinase activity via a PKA-dependent pathway. J Biol Chem 282: 34693-34699, 2007.

5. Liu SK, Smith CA, Arnold R, Kiefer F and McGlade CJ: The adaptor protein Gads (Grb2-related adaptor downstream of Shc) is implicated in coupling hemopoietic progenitor kinase-1 to the activated TCR. J Immunol 165: 1417-1426, 2000.

6. Shui JW, Boomer JS, Han J, Xu J, Dement GA, Zhou G and Tan TH: Hematopoietic progenitor kinase 1 negatively regulates $\mathrm{T}$ cell receptor signaling and $\mathrm{T}$ cell-mediated immune responses. Nat Immunol 8: 84-91, 2007.

7. Brenner D, Golks A, Kiefer F, Krammer PH and Arnold R: Activation or suppression of NFkappaB by HPK1 determines sensitivity to activation-induced cell death. EMBO J 24: 4279-4290, 2005.

8. Brenner D, Golks A, Becker M, Müller W, Frey CR, Novak R, Melamed D, Kiefer F, Krammer PH and Arnold R: Caspase-cleaved HPK1 induces CD95L-independent activation-induced cell death in T and B lymphocytes. Blood 110 : 3968-3977, 2007

9. Ling P, Meyer CF, Redmond LP, Shui JW, Davis B, Rich RR, Hu MC, Wange RL and Tan TH: Involvement of hematopoietic progenitor kinase 1 in T cell receptor signaling. J Biol Chem 276 18908-18914, 2001.

10. Arnold R, Patzak IM, Neuhaus B, Vancauwenbergh S, Veillette A, Van Lint J and Kiefer F: Activation of hematopoietic progenitor kinase 1 involves relocation, autophosphorylation, and transphosphorylation by protein kinase D1. Mol Cell Biol 25: 2364-2383, 2005.

11. Alzabin S, Pyarajan S, Yee H, Kiefer F, Suzuki A, Burakoff S and Sawasdikosol S: Hematopoietic progenitor kinase 1 is a critical component of prostaglandin E2-mediated suppression of the anti-tumor immune response. Cancer Immunol Immunother 59: 419-429, 2010

12. Königsberger S, Peckl-Schmid D, Zaborsky N, Patzak I, Kiefer F and Achatz G: HPK1 associates with SKAP-HOM to negatively regulate Rap1-mediated B-lymphocyte adhesion. PLoS One 5: pii: e12468, 2010.

13. Anafi M, Kiefer F, Gish GD, Mbamalu G, Iscove NN and Pawson T: SH2/SH3 adaptor proteins can link tyrosine kinases to a Ste20-related protein kinase, HPK1. J Biol Chem 272: 27804-27811, 1997.

14. Ling P, Yao Z, Meyer CF, Wang XS, Oehrl W, Feller SM and Tan TH: Interaction of hematopoietic progenitor kinase 1 with adapter proteins Crk and CrkL leads to synergistic activation of c-Jun N-terminal kinase. Mol Cell Biol 19: 1359-1368, 1999.
15. Sawasdikosol S, Russo KM and Burakoff SJ: Hematopoietic progenitor kinase 1 (HPK1) negatively regulates prostaglandin E2-induced fos gene transcription. Blood 101: 3687-3689, 2003.

16. Zhou G, Lee SC, Yao Z and Tan TH: Hematopoietic progenitor kinase 1 is a component of transforming growth factor beta-induced c-Jun N-terminal kinase signaling cascade. J Biol Chem 274: 13133-13138, 1999.

17. Wang W, Zhou G, Hu MC, Yao Z and Tan TH: Activation of the hematopoietic progenitor kinase-1 (HPK1)-dependent, stress-activated c-Jun N-terminal kinase (JNK) pathway by transforming growth factor beta (TGF-beta)-activated kinase (TAK1), a kinase mediator of TGF beta signal transduction. J Biol Chem 272: 22771-22775, 1997.

18. Nagata Y, Kiefer F, Watanabe T and Todokoro K: Activation of hematopoietic progenitor kinase-1 by erythropoietin. Blood 93: 3347-3354, 1999.

19. Arnold R, Frey CR, Müller W, Brenner D, Krammer PH and Kiefer F: Sustained JNK signaling by proteolytically processed HPK1 mediates IL-3 independent survival during monocytic differentiation. Cell Death Differ 14: 568-575, 2007.

20. Chen YR and Tan TH: Inhibition of the c-Jun N-terminal kinase (JNK) signaling pathway by curcumin. Oncogene 17: 173-178, 1998.

21. Balakireva M, Rossé C, Langevin J, Chien YC, Gho M, Gonzy-Treboul G, Voegeling-Lemaire S, Aresta S, Lepesant JA, Bellaiche Y, et al: The Ral/exocyst effector complex counters c-Jun N-terminal kinase-dependent apoptosis in Drosophila melanogaster. Mol Cell Biol 26: 8953-8963, 2006.

22. Ito $Y$, Pandey $P$, Sathyanarayana $P$, Ling $P$, Rana A, Weichselbaum R, Tan TH, Kufe D and Kharbanda S: Interaction of hematopoietic progenitor kinase 1 and c-Abl tyrosine kinase in response to genotoxic stress. J Biol Chem 276: 18130-18138, 2001.

23. Hu MC, Wang Yp, Qiu WR, Mikhail A, Meyer CF and Tan TH: Hematopoietic progenitor kinase-1 (HPK1) stress response signaling pathway activates IkappaB kinases (IKK-alpha/beta) and IKK-beta is a developmentally regulated protein kinase. Oncogene 18: 5514-5524, 1999.

24. Chen YR, Meyer CF, Ahmed B, Yao Z and Tan TH: Caspase-mediated cleavage and functional changes of hematopoietic progenitor kinase 1 (HPK1). Oncogene 18: 7370-7377, 1999.

25. Arnold R, Liou J, Drexler HC, Weiss A and Kiefer F: Caspase-mediated cleavage of hematopoietic progenitor kinase 1 (HPK1) converts an activator of NFkappaB into an inhibitor of NFkappaB. J Biol Chem 276: 14675-14684, 2001.

26. Schulze-Luehrmann J, Santner-Nanan B, Jha MK, Schimpl A, Avots A and Serfling E: Hematopoietic progenitor kinase 1 supports apoptosis of T lymphocytes. Blood 100: 954-960, 2002.

27. Chen YR and Tan TH: The c-Jun N-terminal kinase pathway and apoptotic signaling (Review). Int J Oncol 16: 651-662, 2000.

28. Brenner D, Krammer PH and Arnold R: Concepts of activated T cell death. Crit Rev Oncol Hematol 66: 52-64, 2008.

29. Krammer PH, Arnold R and Lavrik IN: Life and death in peripheral T cells. Nat Rev Immunol 7: 532-542, 2007.

30. Wang H, Song X, Logsdon C, Zhou G, Evans DB, Abbruzzese JL, Hamilton SR, Tan TH and Wang H: Proteasome-mediated degradation and functions of hematopoietic progenitor kinase 1 in pancreatic cancer. Cancer Res 69: 1063-1070, 2009.

31. Zhang Q, Long H, Liao J, Zhao M, Liang G, Wu X, Zhang P, Ding S, Luo S and Lu Q: Inhibited expression of hematopoietic progenitor kinase 1 associated with loss of jumonji domain containing 3 promoter binding contributes to autoimmunity in systemic lupus erythematosus. J Autoimmun 37: 180-189, 2011.

32. Wang X, Li JP, Kuo HK, Chiu LL, Dement GA, Lan JL, Chen DY, Yang CY, Hu H and Tan TH: Down-regulation of B cell receptor signaling by hematopoietic progenitor kinase 1 (HPK1)-mediated phosphorylation and ubiquitination of activated B cell linker protein (BLNK). J Biol Chem 287: 11037-11048, 2012.

33. Stoeckman AK, Baechler EC, Ortmann WA, Behrens TW, Michet CJ and Peterson EJ: A distinct inflammatory gene expression profile in patients with psoriatic arthritis. Genes Immun 7: 583-591, 2006

34. Batliwalla FM, Li W, Ritchlin CT, Xiao X, Brenner M, Laragione T, Shao T, Durham R, Kemshetti S, Schwarz E, et al: Microarray analyses of peripheral blood cells identifies unique gene expression signature in psoriatic arthritis. Mol Med 11: 21-29, 2005. 
35. Chen-Deutsch X, Kutner A, Harrison JS and Studzinski GP: The pan-caspase inhibitor Q-VD-OPh has anti-leukemia effects and can interact with vitamin D analogs to increase HPK1 signaling in AML cells. Leuk Res 36: 884-888, 2012.

36. Chen-Deutsch X and Studzinski GP: Dual role of hematopoietic progenitor kinase 1 (HPK1) as a positive regulator of $1 \alpha, 25$-dihydroxyvitamin D-induced differentiation and cell cycle arrest of AML cells and as a mediator of vitamin D resistance. Cell Cycle 11: 1364-1373, 2012.

37. Wang Y, Luo H, Li Y, Chen T, Wu S and Yang L: hsa-miR-96 up-regulates MAP4K1 and IRS1 and may function as a promising diagnostic marker in human bladder urothelial carcinomas. Mol Med Rep 5: 260-265, 2012.

38. Qian Y, Takeuchi S, Dugu L, Tsuji G, Xie L, Nakahara T, Takahara M, Moroi Y, Tu YT and Furue M: Hematopoietic progenitor kinase 1, mitogen-activated protein/extracellular signal-related protein kinase kinase kinase 1 and phosphomitogen-activated protein kinase kinase 4 are overexpressed in extramammary Paget disease. Am J Dermatopathol 33: 681-686, 2011.

39. Yang HS, Matthews CP, Clair T, Wang Q, Baker AR, Li CC Tan TH and Colburn NH: Tumorigenesis suppressor Pded 4 down-regulates mitogen-activated protein kinase kinase kinase kinase 1 expression to suppress colon carcinoma cell invasion. Mol Cell Biol 26: 1297-1306, 2006.

40. Sawasdikosol S, Zha R, Yang B and Burakoff S: HPK1 as a novel target for cancer immunotherapy. Immunol Res 54: 262-265, 2012.

41. Jakob SM, Pick R, Brechtefeld D, Nussbaum C, Kiefer F, Sperandio $\mathrm{M}$ and Walzog B: Hematopoietic progenitor kinase 1 (HPK1) is required for LFA-1-mediated neutrophil recruitment during the acute inflammatory response. Blood 121: 4184-4194, 2013.

42. Geisberger R, Prlic M, Achatz-Straussberger G, Oberndorfer I, Luger E, Lamers M, Crameri R, Appenzeller U, Wienands J, Breitenbach $\mathrm{M}$, et al: Phage display based cloning of proteins interacting with the cytoplasmic tail of membrane immunoglobulins. Dev Immunol 9: 127-134, 2002.

43. Buday L: Membrane-targeting of signalling molecules by $\mathrm{SH} 2 / \mathrm{SH} 3$ domain-containing adaptor proteins. Biochim Biophys Acta 1422: 187-204, 1999

44. Wilkinson B, Wang $\mathrm{H}$ and Rudd CE: Positive and negative adaptors in T-cell signalling. Immunology 111: 368-374, 2004

45. Ma W, Xia C, Ling P, Qiu M, Luo Y, Tan TH and Liu M: Leukocyte-specific adaptor protein Grap2 interacts with hematopoietic progenitor kinase 1 (HPK1) to activate JNK signaling pathway in T lymphocytes. Oncogene 20: 1703-1714, 2001.

46. Han A, Saijo K, Mecklenbräuker I, Tarakhovsky A and Nussenzweig MC: Bam32 links the B cell receptor to ERK and JNK and mediates B cell proliferation but not survival. Immunity 19: 621-632, 2003

47. Boomer JS and Tan TH: Functional interactions of HPK1 with adaptor proteins. J Cell Biochem 95: 34-44, 2005.

48. Thome M, Charton JE, Pelzer C and Hailfinger S: Antigen receptor signaling to NF-kappaB via CARMA1, BCL10, and MALT1. Cold Spring Harb Perspect Biol 2: a003004, 2010.

49. Brenner D, Brechmann M, Röhling S, Tapernoux M, Mock T, Winter D, Lehmann WD, Kiefer F, Thome M, Krammer PH and Röhling S: Phosphorylation of CARMA1 by HPK1 is critical for NF-kappaB activation in T cells. Proc Natl Acad Sci USA 106 14508-14513, 2009.

50. Chen YR, Kori R, John B and Tan TH: Caspase-mediated cleavage of actin-binding and SH3-domain-containing proteins cortactin, HS1, and HIP-55 during apoptosis. Biochem Biophys Res Commun 288: 981-989, 2001.

51. Patzak IM, Königsberger S, Suzuki A, Mak TW and Kiefer F: HPK1 competes with ADAP for SLP-76 binding and via Rap1 negatively affects T-cell adhesion. Eur J Immunol 40: 3220-3225, 2010.

52. Ruzza P, Siligardi G, Donella-Deana A, Calderan A, Hussain R, Rubini C, Cesaro L, Osler A, Guiotto A, Pinna LA and Borin G: 4-Fluoroproline derivative peptides: Effect on PPII conformation and SH3 affinity. J Pept Sci 12: 462-471, 2006

53. Le Bras S, Foucault I, Foussat A, Brignone C, Acuto O and Deckert M: Recruitment of the actin-binding protein HIP-55 to the immunological synapse regulates $\mathrm{T}$ cell receptor signaling and endocytosis. J Biol Chem 279: 15550-15560, 2004.

54. Han J, Shui JW, Zhang X, Zheng B, Han S and Tan TH: HIP-55 is important for T-cell proliferation, cytokine production, and immune responses. Mol Cell Biol 25: 6869-6878, 2005.

55. Han J, Kori R, Shui JW, Chen YR, Yao Z and Tan TH: The SH3 domain-containing adaptor HIP-55 mediates c-Jun N-terminal kinase activation in $\mathrm{T}$ cell receptor signaling. J Biol Chem 278 : $52195-52202,2003$
56. Ensenat D, Yao Z, Wang XS, Kori R, Zhou G, Lee SC and Tan TH: A novel src homology 3 domain-containing adaptor protein, HIP-55, that interacts with hematopoietic progenitor kinase 1. J Biol Chem 274: 33945-33950, 1999.

57. Liou J, Kiefer F, Dang A, Hashimoto A, Cobb MH, Kurosaki T and Weiss A: HPK1 is activated by lymphocyte antigen receptors and negatively regulates AP-1. Immunity 12: 399-408, 2000.

58. Oehrl W, Kardinal C, Ruf S, Adermann K, Groffen J, Feng GS, Blenis J, Tan TH and Feller SM: The germinal center kinase (GCK)-related protein kinases HPK1 and KHS are candidates for highly selective signal transducers of Crk family adapter proteins. Oncogene 17: 1893-1901, 1998.

59. Lewitzky M, Harkiolaki M, Domart MC, Jones EY and Feller SM: Mona/Gads SH3C binding to hematopoietic progenitor kinase 1 (HPK1) combines an atypical SH3 binding motif, R/KXXK, with a classical PXXP motif embedded in a polyproline type II (PPII) helix. J Biol Chem 279: 28724-28732, 2004.

60. Shen R, Ouyang YB, Qu CK, Alonso A, Sperzel L, Mustelin T, Kaplan MH and Feng GS: Grap negatively regulates T-cell receptor-elicited lymphocyte proliferation and interleukin-2 induction. Mol Cell Biol 22: 3230-3236, 2002.

61. Lasserre R, Cuche C, Blecher-Gonen R, Libman E, Biquand E, Danckaert A, Yablonski D, Alcover A and Di Bartolo V: Release of serine/threonine-phosphorylated adaptors from signaling microclusters down-regulates T cell activation. J Cell Biol 195: 839-853, 2011.

62. Sauer K, Liou J, Singh SB, Yablonski D, Weiss A and Perlmutter RM: Hematopoietic progenitor kinase 1 associates physically and functionally with the adaptor proteins B cell linker protein and SLP-76 in lymphocytes. J Biol Chem 276: 45207-45216, 2001.

63. Tsuji S, Okamoto M, Yamada K, Okamoto N, Goitsuka R, Arnold R, Kiefer F and Kitamura D: B cell adaptor containing src homology 2 domain (BASH) links B cell receptor signaling to the activation of hematopoietic progenitor kinase 1. J Exp Med 194: 529-539, 2001.

64. Yu J, Riou C, Davidson D, Minhas R, Robson JD, Julius M, Arnold R, Kiefer F and Veillette A: Synergistic regulation of immunoreceptor signaling by SLP-76-related adaptor Clnk and serine/threonine protein kinase HPK-1. Mol Cell Biol 21: 6102-6112, 2001

65. Burns JC, Corbo E, Degen J, Gohil M, Anterasian C, Schraven B, Koretzky GA, Kliche S and Jordan MS: The SLP-76 Src homology 2 domain is required for T cell development and activation. J Immunol 187: 4459-4466, 2011.

66. Wang X, Li JP, Chiu LL, Lan JL, Chen DY, Boomer J and Tan TH: Attenuation of $\mathrm{T}$ cell receptor signaling by serine phosphorylation-mediated lysine 30 ubiquitination of SLP-76 protein. J Biol Chem 287: 34091-34100, 2012

67. Di Bartolo V, Montagne B, Salek M, Jungwirth B, Carrette F, Fourtane J, Sol-Foulon N, Michel F, Schwartz O, Lehmann WD and Acuto O: A novel pathway down-modulating $\mathrm{T}$ cell activation involves HPK-1-dependent recruitment of 14-3-3 proteins on SLP-76. J Exp Med 204: 681-691, 2007.

68. Utting O, Sedgmen BJ, Watts TH, Shi X, Rottapel R, Iulianella A, Lohnes D and Veillette A: Immune functions in mice lacking Clnk, an SLP-76-related adaptor expressed in a subset of immune cells. Mol Cell Biol 24: 6067-6075, 2004.

69. Girardin SE and Yaniv M: A direct interaction between JNK1 and CrkII is critical for Rac1-induced JNK activation. EMBO J 20: 3437-3446, 2001.

70. Kiefer F, Tibbles LA, Anafi M, Janssen A, Zanke BW, Lassam N, Pawson T, Woodgett JR and Iscove NN: HPK1, a hematopoietic protein kinase activating the SAPK/JNK pathway. EMBO J 15: 7013-7025, 1996

71. Hu MC, Qiu WR, Wang X, Meyer CF and Tan TH: Human HPK1, a novel human hematopoietic progenitor kinase that activates the JNK/SAPK kinase cascade. Genes Dev 10: 2251-2264, 1996.

72. Marshall AJ, Niiro H, Lerner CG, Yun TJ, Thomas S, Disteche CM and Clark EA: A novel B lymphocyte-associated adaptor protein, Bam32, regulates antigen receptor signaling downstream of phosphatidylinositol 3-kinase. J Exp Med 191: 1319-1332, 2000

73. Hehner SP, Hofmann TG, Dienz O, Droge W and Schmitz ML: Tyrosine-phosphorylated Vavl as a point of integration for T-cell receptor- and CD28-mediated activation of JNK, p38, and interleukin-2 transcription. J Biol Chem 275: 18160-18171, 2000. 